\title{
Exosome-derived microRNAs contribute to prostate cancer chemoresistance
}

\author{
JING LI $^{1,2^{*}}$, XIN YANG ${ }^{1,2^{*}}$, HAO GUAN ${ }^{1,2^{*}}$, ATSUSHI MIZOKAMI ${ }^{3}$, EVAN T. KELLER $^{4}$,

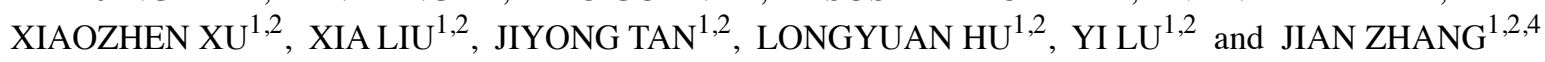 \\ ${ }^{1}$ Key Laboratory of Longevity and Aging-Related Diseases, Ministry of Education, Nanning, Guangxi; \\ ${ }^{2}$ Center for Translational Medicine, Guangxi Medical University, Nanning, Guangxi, P.R. China; \\ ${ }^{3}$ Department of Urology, Kanazawa University, Kanazawa, Japan; ${ }^{4}$ Department of Urology and Pathology, \\ School of Medicine, University of Michigan, Ann Arbor, MI, USA
}

Received March 27, 2016; Accepted May 20, 2016

DOI: $10.3892 /$ ijo.2016.3560

\begin{abstract}
Certain microRNAs (miRNAs) play a key role in cancer cell chemoresistance. However, the pleiotropic functions of exosome-derived miRNAs on developing chemoresistance remain unknown. In the present study, we aimed to construct potential networks of miRNAs, which derived from the exosome of chemoresistant prostate cancer ( $\mathrm{PCa}$ ) cells, with their known target genes using miRNA expression profiling and bioinformatic tools. Global miRNA expression profiles were measured by microarray. Twelve miRNAs were initially selected and validated by qRT-PCR. Known targets of deregulated miRNAs were utilized using DIANA-TarBase database v6.0. The incorporation of deregulated miRNAs and target genes into KEGG pathways were utilized using DIANA-mirPath software. To construct potential miRNA regulatory networks, the overlapping parts of miRNAs and their targer genes from the selected KEGG pathway 'PCa progression (hsa05215)' were visualized by Cytoscape software. We identified 29 deregulated miRNAs, including 19 upregulated and 10 downregulated, in exosome samples derived from two kinds of paclitaxel resistance PCa cells (PC3-TXR and DU145-TXR) compared with their parental cells (PC3 and DU145). The enrichment results of deregulated miRNAs and known target genes showed that a few pathways were correlated with several critical cell signaling pathways. We found that hub hsa-miR3176, -141-3p, -5004-5p, -16-5p, $-3915,-488-3 p,-23 c,-3673$ and -3654 were potential targets to hub gene androgen receptor (AR) and phosphatase and tensin homolog (PTEN). Hub gene T-cell factors/lymphoid
\end{abstract}

Correspondence to: Dr Jian Zhang or Dr Yi Lu, Center for Translational Medicine, Guangxi Medical University, No. 22 Shuangyong Road 1416, Pharmacology and Biomedical Sciences Building, Nanning, Guangxi 530021, P.R. China

E-mail: jianzhang008@hotmail.com

E-mail: luyi5555@hotmail.com

${ }^{*}$ Contributed equally

Key words: exosome, miRNA, prostate cancer, chemoresistance enhancer-binding factors 4 (TCF4) target genes were mainly regulated by hub hsa-miR-32-5, -141-3p, -606, -381 and -429. These results may provide a linkage between PCa chemoresistance and exosome regulatory networks and thus lead us to propose that AR, PTEN and TCF4 genes may be the important genes which are regulated by exosome miRNAs in chemoresistance cancer cells.

\section{Introduction}

Prostate cancer ( $\mathrm{PCa}$ ) is the most frequently diagnosed malignancy and the second leading cause of death for male cancer patients ( $\geq 50$ years old) in western countries (1). Currently, anti-androgen therapy is the first line of treatment for patients diagnosed with PCa. A majority of these patients, however, eventually develop androgen-independent PCa which is highly metastatic and has poor prognosis (2). Taxanes are effective in treating patients diagnosed with androgen-independent PCa. Although clinical trials have proved the initial efficacy of paclitaxel (PTX) in increasing survival in PCa patients (3), about half of patients develop drug resistance. There are a few effective approaches availabe for treating chemoresistant PCa. In addition, the pleotropic functions of exosome-drived miRNAs on developing chemoresistance remain unknown.

Cancer cells release abundant soluble or membranous factors that facilitate their growth and survival. Evidence suggests that small microvesicles with exosomes play a pivotal role in this process. Exosomes are membrane vesicles with a size of 40-100 $\mathrm{nm}$. They contain proteins, mRNAs, microRNAs (miRNAs) and signaling molecules, that reflect the physiological state of their cells of origin and consequently provide a rich source of potential biomarker molecules (4). Exosomes can be taken up by other cells, it is possible that these membrane vesicles could serve as a novel way of intercellular communication and signaling (5). Over the past few years, researchers have revealed that exosomes crosstalk and/or influence major signal pathways in tumor progression, such as hypoxia-driven epithelial-to-mesenchymal transition, angiogenesis and metastasis involving many cell types within the tumor microenvironment (6-8). 
miRNAs are small non-coding RNAs with diverse functions. They can regulate their target genes in a cooperative, combinatorial fashion, where a single miRNA can target multiple mRNA transcripts and distinct miRNAs can target the same mRNA, ensuring control over a large number of cellular functions. Notably, recent studies found that the secretion of miRNAs is also partially mediated through vesicular/ exosome-mediated mehanisms (9). Hence, exosomes play an important role in miRNA regulation. Therefore, we cannot ignore them while studying miRNA targets or designing miRNA-targeted therapeutic strategies.

miRNA profiling through microarrays is an invaluable technique to determine a miRNA signature, which is necessary to figure out the general and specific expression alterations in difference cells or tissue. In the present study, we aimed to explore the exosome derived miRNA contributing to taxanesresistance (TXR) PCa cells compared with their parental cells for elaborating potential effect of exosomal miRNA of drug resistance of PCa cells.

\section{Materials and methods}

Cell culture and preparation of culture medium. Human metastatic PCa cell lines DU145 and PC3 and their PTX resistant versions DU145-TXR and PC3-TXR used in this study were given by Dr Atsushi Mizokami (Kanazawa University, Kanazawa, Japan). All cell lines were maintained in RPMI culture media supplemented with $1 \%$ penicillin/streptomycin and $10 \%$ fetal bovine serum (FBS; Gibco-Life Technologies, Carlsbad, CA, USA) in a humidified incubator containing 5\% $\mathrm{CO}_{2}$ at $37^{\circ} \mathrm{C}$ as previously described (10).

Ultracentrifugation exosome isolation. Exosomes were prepared from the supernatant of cancer cells using differential centrifugations as previously described (11). In brief, supernatant were harvested, centrifuged at $300 \mathrm{x} g$ for $10 \mathrm{~min}$ to eliminate cells and at $16,500 \mathrm{x} \mathrm{g}$ for $20 \mathrm{~min}$ to remove cell debris and particles. Exosomes were pelleted by ultracentrifugation at $120,000 \mathrm{x} \mathrm{g}$ for $70 \mathrm{~min}$ (all steps were performed at $4^{\circ} \mathrm{C}$ ). The exosome pellet was dissolved in nuclease free water and subsequently split and transferred to different RNase free tubes for RNA isolation. Each exosomal sample was harvested from $400 \mathrm{ml}$ cell suspension with $1-4 \times 10^{6}$ cells $/ \mathrm{ml}$. Exosomes were then immediately lysed in respective lysing solution and continued for RNA purification.

Electron microscopy (EM). EM imaging of exosome preparations were performed as follows. Briefly, the pellet from ultracentrifugation was suspended in $50 \mu 1$ of PBS and vortexed briefly and then $5 \mu \mathrm{l}$ was adsorbed to a carbon-coated grid that had been made hydrophilic by a 30 -sec exposure to a glow discharge. Excess liquid was removed with filter paper, and the samples were stained with $0.75 \%$ uranylformate for $30 \mathrm{sec}$. After removing the excess uranylformate, the grid was examined in a Hitachi electron transmission microscope (H-7650).

Western blot analysis. Exosome samples were lysed in RIPA lysis buffer (Sigma-Aldrich, St. Louis, MO, USA) with $1 \mathrm{mM}$ PMSF and protease inhibitor cocktail on ice for $30 \mathrm{~min}$, and then sonicated and quantified using the BCA protein assay kit (Beyotime Institute of Biotechnology, Nanjing, China). Protein fractions were separated by $10 \%$ SDS-PAGE and then were transferred to polyvinylidenedifluoride membranes $(0.22 \mu \mathrm{m}$; Millipore, Billerica, MA, USA). In addition, the membranes blocked with $5 \%(\mathrm{w} / \mathrm{v})$ skim milk powder in Tris-buffered saline with $0.05 \%(\mathrm{v} / \mathrm{v})$ Tween-20 (TTBS) and incubate with mouse anti-TSG101 (1:1,000; Cell Signaling Technology) and mouse anti-Alix (1:1,000; Cell Signaling Technology) in TBST (50 mM Tris, $150 \mathrm{mM} \mathrm{NaCl,} \mathrm{0.05 \%} \mathrm{Tween-20)} \mathrm{with}$ $5 \%$ non-fat dried milk overnight at $4^{\circ} \mathrm{C}$. In addition, they were incubated with horseradish peroxidase secondary antibody for $1 \mathrm{~h}$ at room temperature. Immunodetection was visualized using a chemiluminescent ECL reagent (Beyotime Institute of Biotechnology).

microRNA microarray chip analysis. Total RNA was isolated from exosomal samples using TRIzol reagent (Invitrogen, Carlsbad, CA, USA), according to the manufacturer's instructions. miRNAs of exosome releasing by two pairs of PCa cell lines were detected by microRNA microarrays chip analysis. Microarray analysis was performed on $5 \mu \mathrm{g}$ of total RNA, miRNA expression profiling microarray was completed by using Agilent miRNA microarrays version 2.3 (Agilent Technologies, Santa Clara, CA, USA) and Agilent's miRNA Complete Labeling and Hyb kit (p/n 5190-0456) generates fluorescently labeled miRNAs with a sample input of $100 \mathrm{ng}$ of total RNA. For identification of upregulated and downregulated miRNA, we calculated the miRNA expression ratio of PC3-TXR to PC3 or DU145-TXR to DU145 separately, and then we found the upregulated or downregulated miRNAs in the two pairs of cancer cell lines.

miRNA validation by quantitative real-time PCR ( $q R T-P C R)$. To verify mature miRNA expression levels, qRT-PCR was performed using a High-Specificity qRT-PCR detection kit in conjunction with an ABI 7500 thermal cycler, according to the manufacturer's recommendations. qRT-PCR primer sequences are shown in Table I. We used U6 small nuclear RNA (U6 snRNA) as an endogenous control for normalization. The qRT-PCR results were expressed relative to miRNA expression levels at the threshold cycle $(\mathrm{Ct})$ and were converted to fold changes $\left(2^{-\Delta \Delta C t}\right)$.

Bioinformatic analysis. Target genes of deregulated miRNAs were listed using DIANA-TarBase database v6.0, which include experimentally validated miRNA targets in the literature. To explore the potential function of the whole miRNAs and target genes signature, DIANA-mirPath, a web-based DNA Intelligent Analysis (DIANA)-miRPath v2.0 was used (http:// www.microrna.gr/miRPathv2) to perform enrichment analysis of differentially expressed miRNA gene targets in Kyoto Encyclopedia of Genes and Genomes (KEGG) pathway (12). The pathways were obtained with a P-value $<0.05$ and gene count. 'PCa progression (hsa05215)' in enriched KEGG pathways and related miRNAs were visualized by Cytoscape software (13).

Statistical analysis. Statistical testing was conducted with the assistance of the SPSS 13.0 software. All data are expressed 
Table I. qRT-PCR primer sequences of 12 miRNAs.

\begin{tabular}{llll}
\hline miRNA & Accession & \multicolumn{1}{c}{ miRNA mature sequences } & \multicolumn{1}{c}{ Primer sequence } \\
\hline miR-32-5p & MIMAT0000090 & UAUUGCACAUUACUAAGUUGCA & GTATTGCACATTACTAAGTTGC \\
miR-23c & MIMAT0000418 & AUCACAUUGCCAGUGAUUACCC & GCATCACATTGCCAGTGATTAC \\
miR-3915 & MIMAT0018189 & UUGAGGAAAAGAUGGUCUUAUU & ACGTTGAGGAAAAGATGGTCT \\
miR-451a & MIMAT0001631 & AAACCGUUACCAUUACUGAGUU & GCAAACCGTTACCATTACTGAG \\
miR-1204 & MIMAT0005868 & UCGUGGCCUGGUCUCCAUUAU & TCGTGGCCTGGTCTCCA \\
miR-3607-3p & MIMAT0017985 & ACUGUAAACGCUUUCUGAUG & ACGACTGTAAACGCTTTCTG \\
miR-99b & MIMAT0000689 & CACCCGTAGAACCGACCTTGCG & TCACCCGTAGAACCGACCT \\
miR-16-5p & MIMAT0000069 & UAGCAGCACGUAAAUAUUGGCG & AGTAGCAGCACGTAAATATTG \\
miR-192-3p & MIMAT0004543 & CUGCCAAUUCCAUAGGUCACAG & ACTGCCAATTCCATAGGTC \\
miR-429 & MIMAT0001536 & UAAUACUGUCUGGUAAAACCGU & CGTAATACTGTCTGGTAAAACCG \\
miR-141-3p & MIMAT0000432 & TAACACTGTCTGGTAAAGATGG & ACTAACACTGTCTGGTAAAGATG \\
miR-3176 & MIMAT0015053 & ACTGGCCTGGGACTACCGG & CGACTGGCCTGGGACTAC \\
\hline
\end{tabular}

Table II. miRNAs upregulated in drug resistant cells compared to parent cells.

\begin{tabular}{lcccc}
\hline miRNA symbol & PCT vs. PC (FC) & Log FC & DUT vs. DU (FC) & Log FC \\
\hline hsa-miR-16-5p & 2.05696 & 1.040514 & 2.87645 & 1.524289 \\
hsa-miR-203a & 4.69448 & 2.230965 & 3.44301 & 1.78367 \\
hsa-miR-32-5p & 2.26927 & 1.182228 & 3.63824 & 1.863241 \\
hsa-miR-515-3p & 2.13854 & 1.096626 & 4.00183 & 2.00066 \\
hsa-miR-99b-5p & 3.08952 & 1.627383 & 3.04017 & 1.604152 \\
hsa-miR-590-5p & 2.56255 & 1.35758 & 2.00299 & 1.002155 \\
hsa-miR-451a & 2.9402 & 1.555914 & 2.56517 & 1.359054 \\
hsa-miR-1204 & 4.29763 & 2.103541 & 2.36563 & 1.242224 \\
hsa-miR-4291 & 4.46956 & 2.160133 & 2.43354 & 1.283056 \\
hsa-miR-3673 & 2.0115 & 1.008272 & 6.23188 & 2.639667 \\
hsa-miR-23c & 4.93221 & 2.302234 & 2.41799 & 1.273808 \\
hsa-miR-3654 & 5.79925 & 2.535866 & 2.86458 & 1.518324 \\
hsa-miR-3607-3p & 2.29986 & 1.201546 & 84.6873 & 6.404074 \\
hsa-miR-3915 & 13.0908 & 3.710481 & 2.50603 & 1.325404 \\
hsa-miR-4716-3p & 2.63367 & 1.397075 & 2.78028 & 1.47523 \\
hsa-miR-4722-5p & 2.07909 & 1.055952 & 2.06061 & 1.043071 \\
hsa-miR-488-3p & 4.92981 & 2.301532 & 7.65926 & 2.937205 \\
hsa-miR-4669 & 2.07631 & 1.054022 & 2.58033 & 1.367556 \\
hsa-miR-5004-5p & 2.11074 & 1.077749 & 4.03321 & 2.011929 \\
\hline
\end{tabular}

as means \pm standard deviation (SD). All miRNAs in exosome derived from drug-resistant PCa cells and their parental cells were compared using a t-test to define differentially expressed miRNAs. Results were considered significant when P-values were $<0.05$.

\section{Results}

Confirmation of exosomal vesicles isolated from PCa cell culture supernatant. The enrichment of typical exosome specific marker proteins such as Alix and TSG101 were assessed using western blot analysis (Fig. 1A). In addition, morphological analysis of the exosome using EM revealed a heterogeneous population of vesicles comprising round- shaped 40-100 $\mathrm{nm}$ diameter vesicles, consistent with exosome (Fig. 1B and C).

Altered miRNA expression between two pairs of cancer cells. To identify differentially expressed miRNA between two pairs of cancer cells, we used GenomeStudio software to select probe-expressed miRNAs (probe signals have significant differences in the background) and chose the miRNAs with the expression ratio (PC3-TXR to PC3 or DU145-TXR to DU145) $>2.0$ (as upregulated miRNA) or $<0.5$ (as downregulated miRNA). From the differentially expressed miRNAs, we selected 19 upregulated miRNAs (Table II) and 10 downregulated miRNAs (Table III) which may contribute to $\mathrm{PCa}$ chemoresistance. 
A
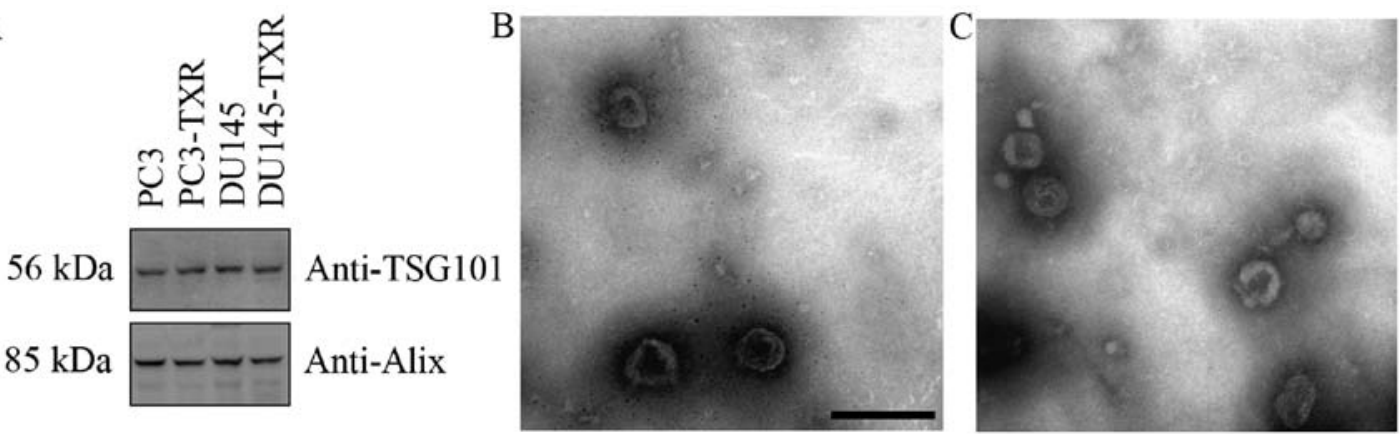

Figure 1. Confirmation of exosomal vesicles isolated from PCa cell culture supernatant. Western blot analysis demonstrated significant enrichment of exosomal marker proteins in the pellet of supernatant from 4 kinds of cells after ultracentrifugation (A). Electron micrograph (EM) of isolated exosomes in the supernatant from PC3 (B) and DU145 (C). The bar, $200 \mathrm{~nm}$.
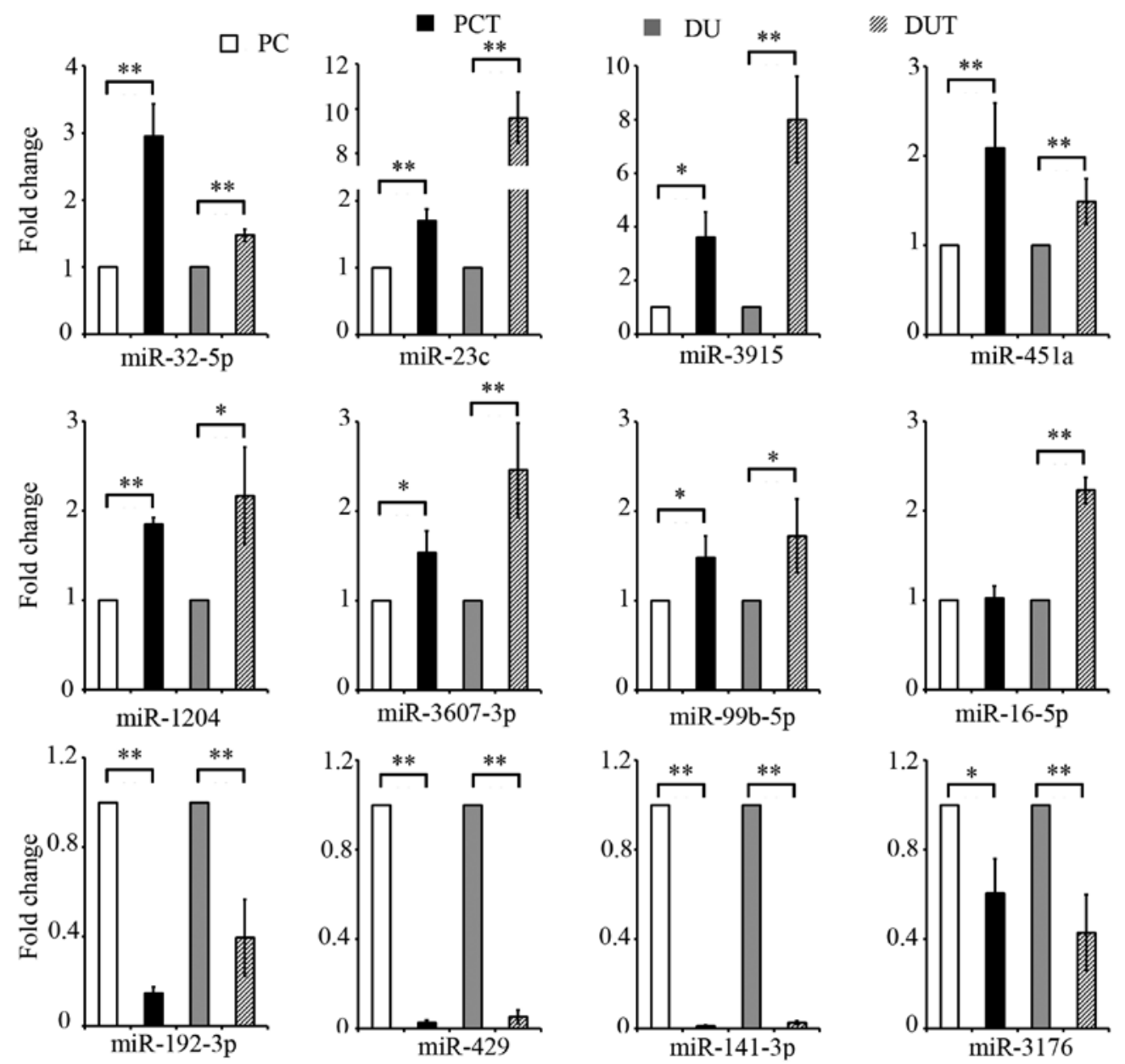

Figure 2. qRT-PCR validation of microarray data. PC, miRNA expression of exosomes secreted from PC3 cells; PCT, miRNA expression of exosomes secreted from PC3-TXR cells; DU, miRNA expression of exosomes secreted from DU145 cells; DUT, miRNA expression of exosomes secreted from DU145-TXR cells. All quantitative data are presented as the mean $\pm \mathrm{SD} ;(\mathrm{n}=3) .{ }^{*} \mathrm{P}<0.05 ;{ }^{* *} \mathrm{P}<0.01$ compared with $\mathrm{PC} 3$ or DU145.

miRNA microarray results were validated by $q R T-P C R$. For validation using qRT-PCR, we chose 8 miRNAs that were upregulated and 4 miRNAs that were downregulated by at least 3 standard deviations in exosome derived from drug-resistant PCa cells compared to those of their parental cells. After normalization with U6 snRNA as the endogenous control, the qRT-PCR data confirmed significant expression $(\mathrm{P}<0.05)$ of all miRNAs in all samples except for miRNA-16 expression in the ratio of PC3-TXR to PC3 cells (Fig. 2).
KEGG pathway analysis. From the differentially expressed miRNAs, we selected 19 upregulated miRNAs (Table II) and 10 downregulated miRNAs (Table III) to predicte target genes using by DIANA-Tarbase v6.0 databases for target analysis (Table IV). Following the addition of upregulated miRNAs, the DIANA-miRPath v2.0 identified top 10 important signaling pathways (Table $\mathrm{V})$ as significantly enriched $(\mathrm{P}<0.05)$. In addition to $\mathrm{PCa}$ and ErbB signaling pathway, insulin signaling pathway and PI3K-Akt signaling pathway were among the 
Table III. miRNAs downregulated in drug resistant cells compared to parent cells.

\begin{tabular}{|c|c|c|c|c|c|}
\hline & miRNA symbol & PCT vs. PC (FC) & $\log \mathrm{FC}$ & DUT vs. DU (FC) & $\log \mathrm{FC}$ \\
\hline 1 & hsa-miR-141-3p & -2.881124063 & -1.526632 & -2.133284129 & -1.093076 \\
\hline 2 & hsa-miR-429 & -17.98522167 & -4.16874 & -3.115885179 & -1.639642 \\
\hline 3 & hsa-miR-192-5p & -2.65166288 & -1.406897 & -2.436187999 & -1.284625 \\
\hline 4 & hsa-miR-192-3p & -5.965686275 & -2.576688 & -2.431876456 & -1.28207 \\
\hline 5 & hsa-miR-606 & -2.791088936 & -1.480828 & -2.155900291 & -1.10829 \\
\hline 6 & hsa-miR-3176 & -4.066972836 & -2.023955 & -2.471960338 & -1.305656 \\
\hline 7 & hsa-miR-1224-3p & -2.073545435 & -1.0521 & -2.843015695 & -1.507422 \\
\hline 8 & hsa-miR-381-3p & -2.563924725 & -1.358354 & -2.769265562 & -1.469503 \\
\hline 9 & hsa-miR-933 & -4.804709053 & -2.264449 & -2.477625641 & -1.308958 \\
\hline 10 & hsa-miR-34b-3p & -3.828528334 & -1.93679 & -2.636757694 & -1.398765 \\
\hline
\end{tabular}

Table IV. Number of known targets of deregulated miRNAs from DIANA microT-CDS.

\begin{tabular}{|c|c|c|c|c|}
\hline & \multicolumn{2}{|c|}{ Upregulated miRNA } & \multicolumn{2}{|c|}{ Downregulated miRNA } \\
\hline & miRNA symbol & Number of target genes & miRNA symbol & Number of target genes \\
\hline 1 & hsa-miR-16-5p & 419 & hsa-miR-141-3p & 308 \\
\hline 2 & hsa-miR-203a & 355 & hsa-mi-429 & 148 \\
\hline 3 & hsa-miR-32-5p & 289 & hsa-miR-192-5p & 39 \\
\hline 4 & hsa-miR-515-3p & 58 & hsa-miR-192-3p & 43 \\
\hline 5 & hsa-miR-99b-5p & 10 & hsa-miR-606 & 76 \\
\hline 6 & hsa-miR-590-5p & 115 & hsa-miR-3176 & 24 \\
\hline 7 & hsa-miR-451a & 9 & hsa-miR-1224-3p & 46 \\
\hline 8 & hsa-miR-1204 & 2 & hsa-miR-381-3p & 268 \\
\hline 9 & hsa-miR-4291 & 121 & hsa-miR-933 & 3 \\
\hline 10 & hsa-miR-3673 & 225 & hsa-miR-34b-3p & 118 \\
\hline 11 & hsa-miR-23c & 192 & & \\
\hline 12 & hsa-miR-3654 & 39 & & \\
\hline 13 & hsa-miR-3607-3p & 304 & & \\
\hline 14 & hsa-miR-3915 & 101 & & \\
\hline 15 & hsa-miR-4716-3p & 11 & & \\
\hline 16 & hsa-miR-4722-5p & 73 & & \\
\hline 17 & hsa-miR-488-3p & 117 & & \\
\hline 18 & hsa-miR-4669 & 2 & & \\
\hline 19 & hsa-miR-5004-5p & 43 & & \\
\hline
\end{tabular}

Table V. Top 10 important pathways of upregulated miRNA in drug resistant cells from DIANA miRPath v.2.0.

\begin{tabular}{|c|c|c|c|c|}
\hline & KEGG pathway & P-value & Genes & miRNAs \\
\hline 1 & ErbB signaling pathway (hsa04012) & $3.40 \mathrm{E}-23$ & 45 & 18 \\
\hline 2 & Long-term potentiation (hsa04720) & $2.63 \mathrm{E}-22$ & 35 & 16 \\
\hline 3 & Insulin signaling pathway (hsa04910) & $4.36 \mathrm{E}-20$ & 59 & 17 \\
\hline 4 & Prostate cancer (hsa05215) & $1.52 \mathrm{E}-19$ & 41 & 15 \\
\hline 5 & PI3K-Akt signaling pathway (hsa04151) & $1.93 \mathrm{E}-19$ & 121 & 17 \\
\hline 6 & Wnt signaling pathway (hsa04310) & $1.86 \mathrm{E}-18$ & 66 & 16 \\
\hline 7 & mTOR signaling pathway (hsa04150) & $5.12 \mathrm{E}-18$ & 32 & 15 \\
\hline 8 & Focal adhesion (hsa04510) & $2.89 \mathrm{E}-17$ & 78 & 17 \\
\hline 9 & Regulation of actin cytoskeleton (hsa04810) & $2.73 \mathrm{E}-15$ & 79 & 17 \\
\hline 10 & Neurotrophin signaling pathway (hsa04722) & $1.23 \mathrm{E}-13$ & 50 & 17 \\
\hline
\end{tabular}


Table VI. TOP 10 important pathways of downregulated miRNA in drug resistant cell from DIANA miRPath v.2.0.

\begin{tabular}{|c|c|c|c|c|}
\hline & KEGG pathway & P-value & Genes & miRNAs \\
\hline 1 & Focal adhesion (hsa04510) & $1.91 \mathrm{E}-16$ & 61 & 8 \\
\hline 2 & Regulation of actin cytoskeleton (hsa04810) & $2.71 \mathrm{E}-15$ & 62 & 9 \\
\hline 3 & TGF- $\beta$ signaling pathway (hsa04350) & $1.76 \mathrm{E}-14$ & 30 & 7 \\
\hline 4 & Ubiquitin mediated proteolysis (hsa04120) & $1.76 \mathrm{E}-14$ & 44 & 9 \\
\hline 5 & ErbB signaling pathway (hsa04012) & $1.65 \mathrm{E}-13$ & 31 & 8 \\
\hline 6 & Axon guidance (hsa04360) & $1.30 \mathrm{E}-10$ & 39 & 8 \\
\hline 7 & Gap junction (hsa04540) & $2.05 \mathrm{E}-10$ & 31 & 9 \\
\hline 8 & Prostate cancer (hsa05215) & $2.50 \mathrm{E}-10$ & 28 & 8 \\
\hline 9 & Pathways in cancer (hsa05200) & $2.50 \mathrm{E}-10$ & 86 & 9 \\
\hline 10 & PI3K-Akt signaling pathway (hsa04151) & 4.73E-09 & 78 & 9 \\
\hline
\end{tabular}

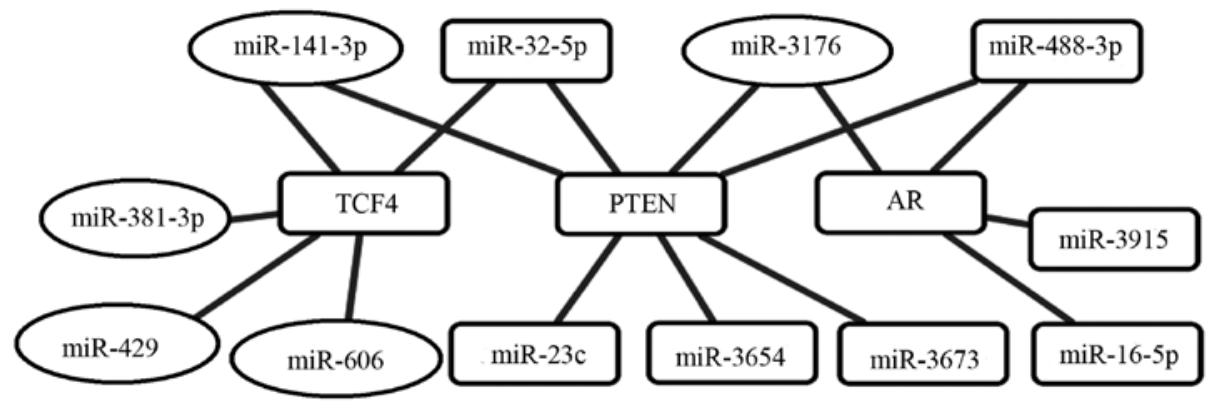

Figure 3. Cytoscape representation of network of related miRNAs and target genes from 'PCa progression (hsa05215)' in KEGG pathways of DIANAmiRPath. Rectangle, upregulated miRNA and oval, downregulated miRNA.

top 5 associated pathways (Table V). Similarly to the downregulated miRNAs, significant enrichment was seen in top 10 important signaling pathways that were mainly influenced by 10 miRNAs (Table VI). Among these, the top 10 important pathways included those involved in focal adhesion, regulation of actin cytoskeleton, TGF- $\beta$ signaling pathway, ubiquitin mediated proteolysis, ErbB signaling pathway (Table VI).

miRNA regulated gene networks associated with PCa chemoresistance. The pathway of 'PCa progression (hsa05215)' category from enriched KEGG pathways was selected for further investigation of the miRNA regulatory networks in PCa (data not shown). Based on these data, the overlapping parts of tow pathways were visualized by Cytoscape software (Fig. 3). We determined hub AR and PTEN target genes mainly regulated by upregulated miRNAs (hsa-miR-16-5p, $-23 c,-32-5 p,-3915,-5004-5 p,-488-3 p,-3673$ and -3654$)$ and downregulated miRNAs (hsa-miR-3176 and -141-3p). Additionally, hub TCF4 target genes mainly regulated by upregulated miRNAs (hsa-miR-32-5p) and downregulated miRNAs (hsa-miR-141-3p, -606, -381 and -429).

\section{Discussion}

miRNAs are a kind of regulatory RNAs that function primarily by targeting specific mRNAs for degradation or inhibition of translation and, thus, decrease the expression of the target protein, and their role in tumor development would be through the regulation of their target protein genes (14). Many studies have shown that miRNAs tend to be expressed abnormally in tumor tissues and cells $(15,16)$. miRNAs function as tumor inhibiting or cancerogenic factors in the development of tumors and also have extensive application value for diagnosing and predicting the prognosis of tumors.

Exosomes are topology identical to that of a cell and contain a broad array of biologically active material including proteins, nucleotides, deoxynucleotides and non-coding miRNAs (17). Emerging evidence indicates that exosomes play a key role in tumor-host crosstalk, and exosome secretion, composition, and functional capacity are altered as tumors progress to an aggressive phenotype. In addition to transmitting signals to other cancer cells, the exosomes released by cancer cells can also impact tumor cell growth, metastasis and angiogenesis and generating the cancer microenvironment $(18,19)$.

In this study, we used the microarray technology to search for miRNA with abnormal expression in exosomes released by PTX resistant PCa cells and their parental cells. We identified and selected 29 miRNAs differentially expressed in two kinds of cells, and this expression profiling might provide a useful clue for indepth research of PCa. In the present study, miR-203 was highly expressed in exosome derived from chemoresistence PCa cells. Recently, a study found that miR-203 is upregulated in three oxaliplatin (L-OHP)-resistant metastatic colorectal cancer cell (CRC) lines and induces L-OHP resistance in CRCs by negatively regulating ataxia telangiectasia mutated kinase (20). Furthermore, miR-203 promote cisplatin 
resistance through suppression of the suppressor of cytokine signaling 3 (21). miR-451 in this study following qPCR validation demonstrated a significant change in exosome derived from chemoresistance PCa cells as compared to their parental cells. In contrast, miR-451 was downregulated in docetaxelresistant lung adenocarcinoma cells (22). However, miR-451 was upregulated in multidrug-resistant (MDR) human ovarian cancer cell line (A2780DX5) and human cervix carcinoma cell line (KB-3-1), compared with their parental lines. Inversely, treatment of A2780DX5 cells with the antagomirs of miR-451 decreased the expression of P-glycoprotein and MDR1 mRNA (23). Additionally, miR-23a was also upregulated in this study. Inhibition of miR-23a expression increases the sensitivity of drug-resistant ovarian cancer cells to cisplatin possibly by miR-23a targeting genes that causes inhibition of P-gp protein expression (24).

In the present study, we observed that expression of 10 miRNAs decreased significantly in the exosome derived from chemoresistance prostate cancer cells compared to their parental cells. Downregulated expression of miR-141 plays a role in selective resistance to L-OHP and epithelial-mesenchymal transition (EMT) in CRCs during repeated treatments with L-OHP (25). miR-429 were downregulated in drugresistant epithelial ovarian cancer tissues (26). Interestingly, overexpression of miR-429 in ovarian cancer cells (OCI-984) induced morphological, functional and molecular changes consistent with EMT and a concomitant significant increase in the sensitivity of the converted cells to cisplatin (27). In addition, miR-429 upregulation induced apoptosis and suppressed invasion by targeting Bcl-2 and SP-1 in esophageal carcinoma (28). Hence, miR-429 may be a potential cancer therapeutic target. miR-381 were strongly downregulated in MDR cells (K562/ADM) by targeting the 3'-UTR of the MDR1 gene and restoring expression of miR-381 in K562/ADM cells was correlated with reduced expression of the MDR1 gene and its protein product, P-gp and increased drug uptake by the cells (29). Additionally, the ubiquitin-specific protease $2 \mathrm{a}$ (USP2a) induced drug resistance in PCa cells, and inhibition of miR-34b made USP $2 \mathrm{a}^{\mathrm{WT}}$ cells trigger drug resistance via miR-34b-driven c-Myc regulation (30).

Several pathways appeared to be enriched by the upregulated miRNAs. Among 10 important pathways, the pathway which associated with ErbB signaling pathway was found to be influenced by 18 miRNAs, which was predicted to target 45 genes. Homo- or heterodimerization of ErbB receptors activate multiple downstream signaling pathways, which are critically involved in multiple biological consequences and thereby promote tumor initiation and progression. Genetic and/or epigenetic alterations of ErbB pathway genes were detected in $80 \%$ of adenocarcinomas (31). Such as the AKT/ERK signaling pathway, which is the pathway downstream of ErbB, was predicted to be active in taxanes-resistant gastric cancer cell lines (32). In addition, the activation of ErbB3 was mainly through PI-3K/Akt signaling playing a vital role in the progression of castration-resistant $\mathrm{PCa}$ into docetaxel-resistance (33).

Similarly, top 10 important signaling pathways of the downregulated miRNAs were noted. Focal adhesion and the regulation of actin cytoskeleton were among the top 5 associated pathways. Notably, these pathways have been implicated in PCa as suggested by other authors. It has been reported that focal adhesion kinase (FAK) could promote the growth, survival, migration, metastasis and androgen-independence of prostate tumors in vitro and in vivo through the activation of major oncogenic pathways (34). More importantly, it was reported that a potential clinical niche for FAK tyrosine kinase inhibitor (TKI) may be used in patients with PCa to overcome chemoresistance because cotreatment with FAK TKI and docetaxel resulted in an additive attenuation of FAK and Akt phosphorylation and overcame the chemoresistant phenotype in PCa PC3 and DU-145 cells (35). Interestingly, FAK inhibition with VS-6063 overcame YB-1-mediated PTX resistance by an AKT-dependent pathway (36) in ovarian cancer. Additionally, the acquisition of drug resistance in ovarian cancer cells induced an extensive reorganization of the actin cytoskeleton, which governed the cellular mechanical properties, motility, and possibly intracellular drug transportation (37).

PCa development is driven by aberrant androgen signaling via the androgen receptor (AR) activity for growth promotion and apoptosis inhibition. Evidence established that taxane stabilization of microtubules inhibits the AR translocation into the nucleus, thus, preventing the transcriptional activity of AR (38). Additionally, taxanes lead to an increase in forkhead box O (FOXO)1, a transcriptional repressor of AR, consequently resulting in inhibition of ligand-dependent and ligand-independent transcription (39). In this study, several miRNA was overexpressed in exosome of taxanes-resistant prostate cancer cells which might be induced by taxanes and target the AR gene to inhibit its activity. However, PC3 and DU145 are of androgen-independent cell lines, known to possess low AR levels (40). Therefore, our findings may indicate the existence of an AR-independent pathway that may be associated with castration PCa. Most importantly, phosphatase and tensin homolog (PTEN) and T-cell factors/lymphoid enhancer-binding factors (TCFs) were regulated by several miRNAs in the present study. PTEN is a tumor-suppressor gene. Deletion of PTEN frequently resulted in tumorigenesis, including primary glioblastomas, breast and lung cancer $(41,42)$. It was shown that chemoresistance is associated with Beclin-1 and PTEN expression in epithelial ovarian cancers. The status of the functional PTEN/FOXO pathway and the drug bioavailability may be the two key determinants for taxol chemoresistance of CRPC in the clinic (43). In the present study, at least 5 upregulated miRNAs and 2 downregulated miRNAs were regulated by the PTEN gene. Our hypothesis is that PTEN is one of the important genes regulated by several upregulated miRNAs via exosomes secreted by PTX-resistant PCa cells in tumor microenvironment. TCF4 are a major class of transcription factors that control the nuclear response to $\mathrm{Wnt} / \beta$-catenin signaling. Some studies indicated that TCF4 functions to promote cellular proliferation $(44,45)$. TCF4 silencing sensitizes the CRC line to L-OHP as a common chemotherapeutic drug (46). Hereby, TCF4 was related to hsa-miR-606, -381 and -429 . In addition, several downregulated miRNAs could promote TCF4 expression. We proposed that TCF4 is one of the important factors regulated by exosomes in tumor microenvironment. However, the exact regulatory networks remain elusive, and it is hard to estimate the actual false-positive 
rate of bioinformatics tools. Therefore, further experimental studies should be carried out in order to confirm our results.

In conclusion, the present study provided novel information that might contribute to a better understanding of molecular mechanisms as well as biological pathways implicated in progressive PCa chemoresistance. This study showed 29 differentially expressed miRNA that bear the potential molecular marker of insensitive PCa cells through targeting known genes to regulate the pathogenesis of PCa. Particularly, hub genes and miRNAs of our constructed network might be central actors of molecular alterations in PCa. Further bench works are needed to confirm their exact roles.

\section{Acknowledgements}

The present study was supported by the Natural Science Foundation of China (NSFC) Key Project (81130046); the NSFC (81171993; 81272415; 81560505); the Guangxi Projects of China (2013GXNSFEA053004; 2012GXNSFCB053004; 2013GXNSFBA019177; 1355004-5; 201201ZD004; GZPT13-35; 14122008-22; 11-031-05-K2; KY2015YB057; 14-045-12-K2). The authors thank Drs Jiejun Fu and Chunlin Zou for helpful discussions and Ms. Xin Huang for editing.

\section{References}

1. Fendler A, Jung M, Stephan C, Honey RJ, Stewart RJ, Pace KT, Erbersdobler A, Samaan S, Jung K and Yousef GM: miRNAs can predict prostate cancer biochemical relapse and are involved in tumor progression. Int J Oncol 39: 1183-1192, 2011.

2. van Brussel JP and Mickisch GH: Multidrug resistance in prostate cancer. Onkologie 26: 175-181, 2003.

3. Tannock IF, de Wit R, Berry WR, Horti J, Pluzanska A, Chi KN, Oudard S, Théodore C, James ND, Turesson I, et al; TAX 327 Investigators: Docetaxel plus prednisone or mitoxantrone plus prednisone for advanced prostate cancer. N Engl J Med 351: 1502-1512, 2004.

4. Simpson RJ, Lim JW, Moritz RL and Mathivanan S: Exosomes: Proteomic insights and diagnostic potential. Expert Rev Proteomics 6: 267-283, 2009.

5. Camussi G, Deregibus MC, Bruno S, Cantaluppi V and BianconeL: Exosomes/microvesicles as a mechanism of cell-tocell communication. Kidney Int 78: 838-848, 2010.

6. An T, Qin S, Xu Y, Tang Y, Huang Y, Situ B, Inal JM and Zheng L: Exosomes serve as tumour markers for personalized diagnostics owing to their important role in cancer metastasis. J Extracell Vesicles 4: 27522, 2015.

7. Zhang J, Li S, Li L, Li M, Guo C, Yao J and Mi S: Exosome and exosomal microRNA: Trafficking, sorting, and function. Genomics Proteomics Bioinformatics 13: 17-24, 2015.

8. Hannafon BN, Carpenter KJ, Berry WL, Janknecht R, Dooley WC and Ding WQ: Exosome-mediated microRNA signaling from breast cancer cells is altered by the anti-angiogenesis agent docosahexaenoic acid (DHA). Mol Cancer 14: 133, 2015.

9. Yang M, Chen J, Su F, Yu B, Su F, Lin L, Liu Y, Huang JD and Song E: Microvesicles secreted by macrophages shuttle invasionpotentiating microRNAs into breast cancer cells. Mol Cancer 10: 117, 2011.

10. Li F, Danquah M, Singh S, Wu H and Mahato RI: Paclitaxeland lapatinib-loaded lipopolymer micelles overcome multidrug resistance in prostate cancer. Drug Deliv Transl Res 1: 420-428, 2011.

11. Lässer C, Eldh $\mathrm{M}$ and Lötvall J: Isolation and characterization of RNA-containing exosomes. J Vis Exp 9: e3037, 2012.

12. Vlachos IS, Kostoulas N, Vergoulis T, Georgakilas G, Reczko M, Maragkakis M, Paraskevopoulou MD, Prionidis K, Dalamagas T and Hatzigeorgiou AG: DIANA miRPath v.2.0: Investigating the combinatorial effect of microRNAs in pathways. Nucleic Acids Res 40 (W1): W498-504, 2012.
13. Shannon P, Markiel A, Ozier O, Baliga NS, Wang JT, Ramage D, Amin N, Schwikowski B and Ideker T: Cytoscape: A software environment for integrated models of biomolecular interaction networks. Genome Res 13: 2498-2504, 2003.

14. Bian Z, Li LM, Tang R, Hou DX, Chen X, Zhang CY and Zen K: Identification of mouse liver mitochondria-associated miRNAs and their potential biological functions. Cell Res 20: 1076-1078, 2010.

15. Zhang C, Wang C, Chen X, Yang C, Li K, Wang J, Dai J, Hu Z, Zhou X, Chen L, et al: Expression profile of microRNAs in serum: A fingerprint for esophageal squamous cell carcinoma. Clin Chem 56: 1871-1879, 2010.

16. Iorio MV and Croce CM: microRNA involvement in human cancer. Carcinogenesis 33: 1126-1133, 2012.

17. Makino DL, Halbach F and Conti E: The RNA exosome and proteasome: Common principles of degradation control. Nat Rev Mol Cell Biol 14: 654-660, 2013.

18. Janowska-Wieczorek A, Wysoczynski M, Kijowski J, MarquezCurtis L, Machalinski B, Ratajczak J and Ratajczak MZ: Microvesicles derived from activated platelets induce metastasis and angiogenesis in lung cancer. Int J Cancer 113: 752-760, 2005.

19. Kruger S, Abd Elmageed ZY, Hawke DH, Wörner PM, Jansen DA, Abdel-Mageed AB, Alt EU and Izadpanah R: Molecular characterization of exosome-like vesicles from breast cancer cells. BMC Cancer 14: 44, 2014.

20. Zhou Y, Wan G, Spizzo R, Ivan C, Mathur R, Hu X, Ye X, Lu J, Fan F, Xia L, et al: miR-203 induces oxaliplatin resistance in colorectal cancer cells by negatively regulating ATM kinase. Mol Oncol 8: 83-92, 2014.

21. Ru P, Steele R, Hsueh EC and Ray RB: Anti-miR-203 upregulates SOCS3 expression in breast cancer cells and enhances cisplatin chemosensitivity. Genes Cancer 2: 720-727, 2011.

22. Wang R, Chen DQ, Huang JY, Zhang K, Feng B, Pan BZ, Chen J, De W and Chen LB: Acquisition of radioresistance in docetaxel-resistant human lung adenocarcinoma cells is linked with dysregulation of miR-451/c-Myc-survivin/rad-51 signaling. Oncotarget 5: 6113-6129, 2014.

23. Zhu H, Wu H, Liu X, Evans BR, Medina DJ, Liu CG and Yang JM: Role of MicroRNA miR-27a and miR-451 in the regulation of MDR1/P-glycoprotein expression in human cancer cells. Biochem Pharmacol 76: 582-588, 2008.

24. Jin AH, Zhou XP and Zhou FZ: Inhibition of microRNA-23a increases cisplatin sensitivity of ovarian cancer cells: The possible molecular mechanisms. Nan Fang Yi Ke Da Xue Xue Bao 35: 125-128, 2015 (In Chinese).

25. Tanaka S, Hosokawa M, Yonezawa T, Hayashi W, Ueda K and Iwakawa S: Induction of epithelial-mesenchymal transition and down-regulation of miR-200c and miR-141 in oxaliplatinresistant colorectal cancer cells. Biol Pharm Bull 38: 435-440, 2015.

26. Liu L, Zou J, Wang Q, Yin FQ, Zhang W and Li L: Novel microRNAs expression of patients with chemotherapy drugresistant and chemotherapy-sensitive epithelial ovarian cancer. Tumour Biol 35: 7713-7717, 2014.

27. Wang L, Mezencev R, Svajdler M, Benigno BB and McDonald JF: Ectopic over-expression of miR-429 induces mesenchymal-toepithelial transition (MET) and increased drug sensitivity in metastasizing ovarian cancer cells. Gynecol Oncol 134: 96-103, 2014.

28. Liu D, Xia P, Diao D, Cheng Y, Zhang H, Yuan D, Huang C and Dang C: MiRNA-429 suppresses the growth of gastric cancer cells in vitro. J Biomed Res 26: 389-393, 2012.

29. Xu Y, Ohms SJ, Li Z, Wang Q, Gong G, Hu Y, Mao Z, Shannon MF and Fan JY: Changes in the expression of miR-381 and miR-495 are inversely associated with the expression of the MDR1 gene and development of multi-drug resistance. PLoS One 8: e82062, 2013.

30. Benassi B, Marani M, Loda M and Blandino G: USP2a alters chemotherapeutic response by modulating redox. Cell Death Dis 4: e812, 2013.

31. Hoque MO, Brait M, Rosenbaum E, Poeta ML, Pal P, Begum S, Dasgupta S, Carvalho AL, Ahrendt SA, Westra WH, et al: Genetic and epigenetic analysis of erbB signaling pathway genes in lung cancer. J Thorac Oncol 5: 1887-1893, 2010.

32. Wu G, Qin XQ, Guo JJ, Li TY and Chen JH: AKT/ERK activation is associated with gastric cancer cell resistance to paclitaxel. Int J Clin Exp Pathol 7: 1449-1458, 2014. 
33. Jathal MK, Chen L, Mudryj M and Ghosh PM: Targeting ErbB3: the new RTK(id) on the prostate cancer block. Immunol Endocr Metab Agents Med Chem 11: 131-149, 2011.

34. Figel S and Gelman IH: Focal adhesion kinase controls prostate cancer progression via intrinsic kinase and scaffolding functions. Anticancer Agents Med Chem 11: 607-616, 2011.

35. Lee BY, Hochgräfe F, Lin HM, Castillo L, Wu J, Raftery MJ, Martin Shreeve S, Horvath LG and Daly RJ: Phosphoproteomic profiling identifies focal adhesion kinase as a mediator of docetaxel resistance in castrate-resistant prostate cancer. Mol Cancer Ther 13: 190-201, 2014.

36. Kang Y, Hu W, Ivan C, Dalton HJ, Miyake T, Pecot CV, Zand B, Liu T, Huang J, Jennings NB, et al: Role of focal adhesion kinase in regulating YB-1-mediated paclitaxel resistance in ovarian cancer. J Natl Cancer Inst 105: 1485-1495, 2013.

37. Seo YH, Jo YN, Oh YJ and Park S: Nano-mechanical reinforcement in drug-resistant ovarian cancer cells. Biol Pharm Bull 38: 389-395, 2015.

38. Darshan MS, Loftus MS, Thadani-Mulero M, Levy BP, Escuin D, Zhou XK, Gjyrezi A, Chanel-Vos C, Shen R, Tagawa ST, et al: Taxane-induced blockade to nuclear accumulation of the androgen receptor predicts clinical responses in metastatic prostate cancer. Cancer Res 71: 6019-6029, 2011.

39. Gan L, Chen S, Wang Y, Watahiki A, Bohrer L, Sun Z, Wang Y and Huang H: Inhibition of the androgen receptor as a novel mechanism of taxol chemotherapy in prostate cancer. Cancer Res 69: 8386-8394, 2009.
40. Alimirah F, Chen J, Basrawala Z, Xin H and Choubey D: DU-145 and PC-3 human prostate cancer cell lines express androgen receptor: Implications for the androgen receptor functions and regulation. FEBS Lett 580: 2294-2300, 2006.

41. Mizoguchi M, Nutt CL, Mohapatra G and Louis DN: Genetic alterations of phosphoinositide 3-kinase subunit genes in human glioblastomas. Brain Pathol 14: 372-377, 2004.

42. Kohno T, Takahashi M, Manda R and Yokota J: Inactivation of the PTEN/MMAC1/TEP1 gene in human lung cancers. Genes Chromosomes Cancer 22: 152-156, 1998.

43. Jiang $\mathbf{J}$ and Huang H: Targeting the Androgen Receptor by taxol in castration-resistant prostate cancer. Mol Cell Pharmacol 2: $1-5,2010$.

44. van de Wetering M, Sancho E, Verweij C, de Lau W, Oving I, Hurlstone A, van der Horn K, Batlle E, Coudreuse D, Haramis AP, et al: The beta-catenin/TCF-4 complex imposes a crypt progenitor phenotype on colorectal cancer cells. Cell 111: 241-250, 2002

45. Shin HW, Choi H, So D, Kim YI, Cho K4, Chung HJ5, Lee KH6, Chun YS7, Cho CH1, Kang GH, et al: ITF2 prevents activation of the beta-catenin-TCF4 complex in colon cancer cells and levels decrease with tumor progression. Gastroenterology 147: 430-442 e438, 2014.

46. Gheidari F, Bakhshandeh B, Teimoori-Toolabi L, Mehrtash A, Ghadir M and Zeinali S: TCF4 silencing sensitizes the colon cancer cell line to oxaliplatin as a common chemotherapeutic drug. Anticancer Drugs 25: 908-916, 2014. 